\title{
T-SPOT. TB assay and tuberculin skin test for diagnosis and screening of tuberculosis: First report in a Sri lankan population
}

\author{
Champa N Ratnatunga', Vasanthi Thevanesam¹, Dushantha Madegedara², Neranjan LA Dissanayake ${ }^{2}$, \\ K.G.R. Athula Kumara ${ }^{1}$
}

${ }^{1}$ Department of Microbiology, Faculty of Medicine, University of Peradeniya, Kandy, Sri Lanka. ${ }^{2}$ Respiratory Disease Treatment Unit, Teaching Hospital, Kandy, Sri Lanka

Objective: Guidelines encourage evaluation of an Interferon Gamma Release Assay (IGRA) in the local setting, particularly in low/middle income, Bacillus Calmette Guerin (BCG) vaccinated populations where the assays have shown variable utility. The T-SPOT.TB assay and the Tuberculin Skin Test (TST) were compared in diagnosis of active tuberculosis (TB) and in contact screening in an adult Sri Lankan population. Materials and Methods: A prospective study including confirmed TB cases $(n=75)$, controls $(n=-74)$ and close contacts of smear positive cases $(n=27)$ was carried out at the regional Chest Clinic in Kandy district. Clinical history and investigation findings, including TST results were recorded and the T-SPOT. $T B$ (Oxford Immunotec) performed. Results: The presence of diabetes and cavitation were significant predictors of T-SPOT.TB positivity, while TST had no significant clinical predictors. In the diagnosis of active TB, T-SPOT.TB sensitivity was $73.3 \%(95 \% \mathrm{Cl}, 58.6-84.6 \%)$ and a specificity was $72 \%(95 \% \mathrm{Cl}, 62.0-85.5 \%)$ while the TST sensitivity was $70.7 \%(95 \% \mathrm{Cl}$, $54.2-83.3 \%)$ and specificity was $64.1 \%(95 \% \mathrm{Cl}, 49.7-76.5 \%)$. In contact investigation neither test showed an association with exposure level. Cost estimate was LKR 9400.00 per T-SPOT.TB test compared to LKR 200.00 per TST. A high (21\%) indeterminate result rate was seen with the T-SPOT.TB assay. Conclusions: This study did not show any advantage in using T-SPOT.TB over TST in this setting.

Key words: Immunologic tests, Interferon-gamma release tests, Latent tuberculosis infection, Tuberculin test, Tuberculosis

\section{INTRODUCTION}

The Interferon gamma release assays (IGRAs) have been in use for the diagnosis and screening of tuberculosis (TB) for over a decade. Evaluation of their use in high, moderate and low TB burden settings as well as in BCG vaccinated and un-vaccinated populations have shown varied results depending on the commercial assay used, characteristics of the patient population (age, immune status, disease status) and study design (cohort vs case control) $\cdot^{1-6}$ As the tests are functional $\mathrm{T}$ cell assays that test the effector memory $\mathrm{T}$ cell response to the Mycobacterium tuberculosis (MTB) antigens early secretory antigenic target 6
(ESAT-6) and culture filtrate protein 10 (CFP-10), the strength, duration and type of previous antigen exposure, the functionality of the patients T cells as well as numerous other variables intrinsic to the test procedure, affect the final result of the IGRAs. ${ }^{7-9}$ This has led to somewhat variable recommendations in guidelines for IGRA use in different settings. ${ }^{9-12} \mathrm{~A}$ common recommendation is that IGRAs need to be assessed in local conditions and guidelines for use be adapted according to local priorities and needs, particularly in low and middle income countries where the assays have not been extensively evaluated., ${ }^{2,13}$ The century old tuberculin skin test (TST) is the most widely used method for TB screening in these settings. 
Despite of many disadvantages of the $\mathrm{TST}^{14-16}$ it remains in use due to the significant advantage of low cost.

Sri Lanka is a moderate income, moderate TB burden country with a TB incidence rate of 66 cases per 100,000 population. ${ }^{17}$ Mandatory BCG vaccination at birth has been carried out as national policy since 1963 with good immunization coverage rates. The TST is widely used for TB screening in close contacts and is also used as a supportive test in diagnosis of active TB. ${ }^{18}$ Latent TB infection (LTBI) is not routinely treated with isoniazid prophylactic therapy. Smear microscopy and TST carried out island-wide under the national TB control programme remain cornerstones of TB diagnostics.

IGRA testing has been available in private sector laboratories and hospitals in Sri Lanka for several years. Though there is no data on the utility of these tests in the local population either as a diagnostic or screening method, it is being used for both purposes. The assay is expensive and as no local guidelines are available, interpretation of results is not uniform. This study was undertaken to brigde this gap in knowledge. A preliminary study to compare a commercial IGRA and the TST for utility in diagnosis of active TB (as a surrogate for LTBI) and LTBI screening was designed.

\section{MATERIALS AND METHODS}

A prospective study was carried out from December 2012 to November 2013 at the Kandy district chest clinic where 200-300 patients smear positive TB patients are registered each year (includes all TB patients in the Kandy district). Patients from other districts are diagnosed here and referred to local clinics for follow up.

Ethical approval was obtained from the Ethical review committee of the Faculty of Medicine, University of Peradeniya. To evaluate test performance in active TB patients a case-control study design was used. Adult patients registering under the care of the collaborating respiratory physician, with no previous history of $\mathrm{TB}$, who were being investigated for current pulmonary $\mathrm{TB}$ were eligible to participate. Convenience sampling was used, based on availability of resources. Patients were recruited in the study only after informed written consent was obtained. Clinical history, examination, investigation results (including smear microscopy, TST and chest $\mathrm{X}$ ray results) were documented using an interviewer administered questionnaire. Sputum culture for TB was performed in addition to routine smear microscopy for acid fast bacilli (AFB). Cases were defined as patients with microbiologically confirmed (smear and/or culture positive) tuberculosis, and controls were defined as patients with no microbiological or other features (chest X ray) suggestive of tuberculosis. Patients who were microbiologically negative but started on anti tuberculous therapy (ATT) based on other findings were excluded. To evaluate the tests in screening for LTBI a small cohort of close contacts of smear positive patients were recruited. Clinical details including duration and category of exposure, TST result and chest $\mathrm{X}$ ray findings were recorded.

Blood was collected from all study participants and the T-SPOT. TBassay (Oxford Immunotec, Abingdon, UK) was performed according to manufacturer's instructions. Spot forming cell (SFC) counting was done manually. TB culture and T-SPOT.TBassay were performed at the Department of Microbiology, Faculty of Medicine, University of Peradeniya.

As a high number of indeterminate results due to positive control failure were observed with the TSPOT.TB assay, the positive control was tested with a quality controlled phytohaemagglutininmitogen) reagent requested from the manufacturer.

Clinical features associated with disease status, T-SPOT. TBpositivity and TST positivity were evaluated using chi square test and significant predictors were modelled with logistic regression models using type 3 effects. Sensitivity of diagnosing of active TB was assessed. Agreement between tests was evaluated with kappa statistic. Data was analyzed using Minitab 14.1, SAS 9.1.3.

\section{RESULTS}

A total of 187 subjects were recruited. 75 patients classified as cases based on smear microscopy/sputum culture results, 74 classified as controls and 27 close contacts of TB patients were included. 11 patients were excluded as ATT was started without microbiological evidence of TB.

Male to female ratio(M:F) in cases (3.8:1) was significantly greater than that in controls $(1: 1)(\mathrm{p}<0.05)$. Both groups were age matched with a mean age of 48.3 years $(95 \%$ CI 44.9-51.8 years) in cases and 52.4years (95\% CI 48.9-56.0 years) in controls ( $p>0.05)$. Gender ratio in contacts was M:F 1:1.4.This group was significantly younger than the control group $(\mathrm{p}<0.05)$ with a mean age of 43 years ( $95 \%$ CI 37.1- 47.8 years).

Clinical features including duration of cough, haemoptysis, wheeze, smoking, fever, night sweats, loss of appetite, loss of weight, contact history, diabetes mellitus and cavitation on chest X ray were evaluated as predictors. Final logistic 
models showing predictors of disease status (cases), T-SPOT.TBpositivity and TST positivity are shown in Table 1. The models show that while disease status and T-SPOT.TBpositivity have similar predictors (DM and cavitation on chest $\mathrm{X}$ ray), TST positivity is not predicted by any of the clinical features tested.

Test characteristics (sensitivity, specificity, positive predictive value (PPV), and negative predictive value (NPV) of the T-SPOT.TBand the TST in the study population for the diagnosis of active tuberculosis (cases vs. controls) are shown in Table 2.

Both tests had similar sensitivity (T-SPOT.TB 73.5\% vs. TST 70.7\%) and NPVs (T-SPOT.TB76.8\% vs. TST 73.9\%). The point estimate of the specificity of the T-SPOT.TBwas higher than that of the TST (T-SPOT.TB75.4\% vs. TST $64.1 \%$ ) though the difference was not significant in this study. Similar results were seen for the PPV. The wide 95\% $\mathrm{CI}$ of the estimates reflect both the variability seen in test results as well as the small sample size.

Of the total 27 household contacts of smear positive patients who were included, none had evidence of active TB based on chest $\mathrm{X}$ ray and sputum microscopy findings. The number of subjects in each exposure category identified is shown in Table 3 .

Association of T-SPOT.TBand TST results with category of exposure was analyzed with Fishers- exact or Chi-square test as appropriate. T-SPOT.TBwas positive in $25.9 \%$ and negative in $59.2 \%$ of contacts. There was no significant association between T-SPOT.TBpositivity and sleep exposure $(\mathrm{p}=0.376)$, categorical (proximity of) exposure $(p=0.146)$ or number of exposure hours $(p=0.207)$. TST was positive in $53.8 \%$ and negative in $46.2 \%$ of contacts. There was no significant association between sleep exposure $(p=0.821)$, categorical exposure $(p=0.792)$ or number of exposure hours $(\mathrm{p}=0.409)$ with the TST result. Though a lower number of contacts were T-SPOT. TBpositive compared with number who were TST positive, there was also no significant difference in the proportions $(p=0.098)$. All TSPOT-TBcontacts were also TST positive however, all TST positive contacts were not found to be TSPOT-TB positive.

The tests showed moderate concordance $(x=0.4113$, $\mathrm{p}<0.0001)$ for all subjects, controls $(x=0.415, \mathrm{p}=0.005)$ and contacts $(x=0.476, \mathrm{p}=0.009)$ but was poor for cases $(x=0.184, \mathrm{p}=0.305)$. A comparison of TST reading and SFC count in each category are shown in Figures 1,2 and 3.

$83 \%$ of indeterminate results were due to positive control (mitogen) failure $(p<0.001)$. Mitogen failure occurred significantly more often in cases than in controls ( 2 proportions, $\mathrm{p}=0.029$ ) There was no association between indeterminate results in cases and the presence of DM $(p=0.681)$ and no difference in the mean age of subjects with indeterminate results $(p=0.449)$. Repeat testing was

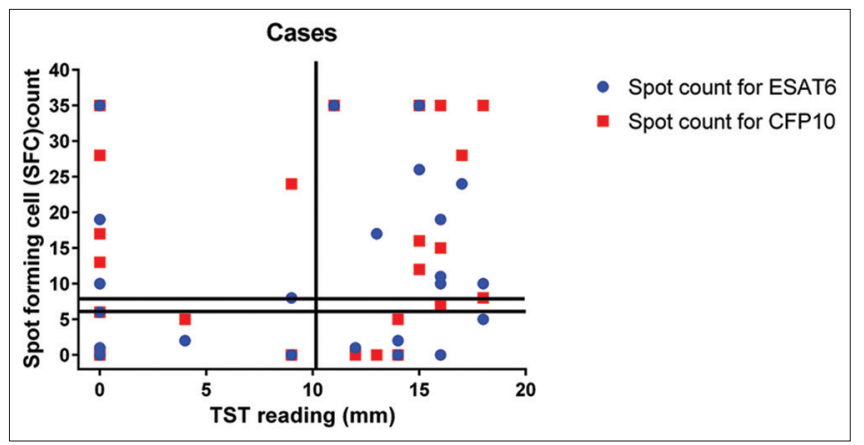

Figure 1: Scatter plots of Tuberculin Skin test reading $(\mathrm{mm})$ vs Spot forming cell (SFC) count for ESAT 6 and CFP10 antigens in cases. Reference lines show $10 \mathrm{~mm}$ cut-off for TST positivity (x axis) and SFC count of 6-8 indicating TSPOT.TB positivity (y axis)

\begin{tabular}{|c|c|c|c|c|c|}
\hline \multirow{2}{*}{$\begin{array}{l}\text { Outcome } \\
\text { (model significance) }\end{array}$} & \multirow[t]{2}{*}{ Predictor } & \multirow[t]{2}{*}{$p$} & \multirow{2}{*}{$\begin{array}{l}\text { Odds } \\
\text { ratio }\end{array}$} & \multicolumn{2}{|c|}{$95 \% \mathrm{Cl}$} \\
\hline & & & & Lower & Upper \\
\hline \multirow[t]{6}{*}{ Disease status $(p<0.0001)$} & Constant & $<0.0001$ & & & \\
\hline & Gender (female to male) & 0.0147 & 0.26 & 0.088 & 0.767 \\
\hline & Loss of appetite & 0.0109 & 4.6 & 1.426 & 15.248 \\
\hline & Loss of weight & 0.069 & 5.16 & 1.569 & 17.021 \\
\hline & $\mathrm{DM}^{*}$ & 0.0038 & 7.29 & 1.897 & 28.021 \\
\hline & Cavitation & $<0.0001$ & 81.7 & 14.485 & 461.167 \\
\hline \multirow[t]{3}{*}{ T-SPOT.TB positivity $(p<0.001)$} & Constant & $<0.001$ & & & \\
\hline & $\mathrm{DM}^{*}$ & 0.016 & 4.3 & 1.3 & 14.0 \\
\hline & Cavitation & $<0.0001$ & 11.9 & 3.7 & 38.8 \\
\hline \multirow[t]{4}{*}{ TST positivity $(p=0.0024)$} & Constant & 0.005 & & & \\
\hline & Fever & 0.057 & 2.47 & 0.97 & 6.26 \\
\hline & $\mathrm{DM}^{*}$ & 0.172 & 2.13 & 0.72 & 6.28 \\
\hline & Cavitation & 0.068 & 2.71 & 0.93 & 7.90 \\
\hline
\end{tabular}




\begin{tabular}{lccccc}
\multicolumn{3}{l}{$\begin{array}{l}\text { Table 2: Test characteristics of T-SPOT.TB and } \\
\text { TST* in diagnosis of active pulmonary TB }\end{array}$} \\
$\begin{array}{lccccc}\text { Test } \\
\text { characteristic }\end{array}$ & \multicolumn{2}{c}{ T-SPOT.TB } & & \multicolumn{2}{c}{ TST } \\
\cline { 2 - 3 } \cline { 5 - 6 } & $\%$ & $95 \% \mathbf{C l}$ & & $\%$ & $\mathbf{9 5 \%} \mathbf{C l}$ \\
\hline Sensitivity & 73.5 & $58.6-84.6$ & & 70.7 & $54.2-83.3$ \\
Specificity & 75.4 & $62.0-85.5$ & & 64.1 & $49.7-76.5$ \\
PPV $^{* *}$ & 72.0 & $57.3-83.3$ & & 60.4 & $45.2-73.8$ \\
NPV $^{* * *}$ & 76.8 & $63.2-86.6$ & & 73.9 & $58.5-85.2$ \\
\hline
\end{tabular}

*TST - Tuberculin skin test, **PPV - Positive predictive value, $* * * N P V$ - Negative predictive value

\begin{tabular}{|c|c|c|c|c|c|}
\hline $\begin{array}{l}\text { Sleeping } \\
\text { exposure }\end{array}$ & $\mathrm{n}$ & $\begin{array}{l}\text { Categorical } \\
\text { exposure }\end{array}$ & $\mathrm{n}$ & $\begin{array}{l}\text { Exposure } \\
\text { hours/day }\end{array}$ & $\mathbf{n}$ \\
\hline Same bedroom & 14 & $\begin{array}{l}\text { Live in the same } \\
\text { house or share } \\
\text { workplace with case }\end{array}$ & 23 & $<=8$ hours & 7 \\
\hline $\begin{array}{l}\text { Same house/ } \\
\text { different house in } \\
\text { same compound }\end{array}$ & 13 & $\begin{array}{l}\text { Same room as } \\
\text { case at least once } \\
\text { a week }\end{array}$ & 4 & $>8$ hours & 20 \\
\hline
\end{tabular}

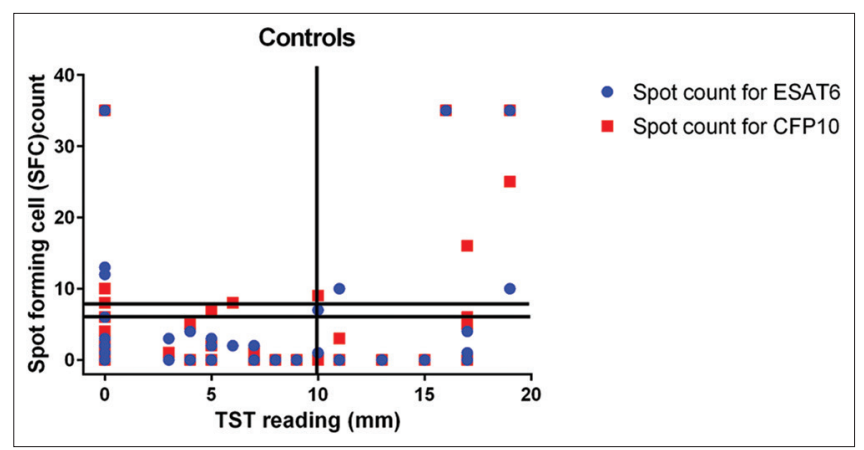

Figure 2: Scatter plots of Tuberculin Skin test reading $(\mathrm{mm})$ vs Spot forming cell (SFC) count for ESAT 6 and CFP10 antigens in contacts. Reference lines show $10 \mathrm{~mm}$ cut-off for TST positivity ( $x$ axis) and SFC count of 6-8 indicating TSPOT.TB positivity (y axis)

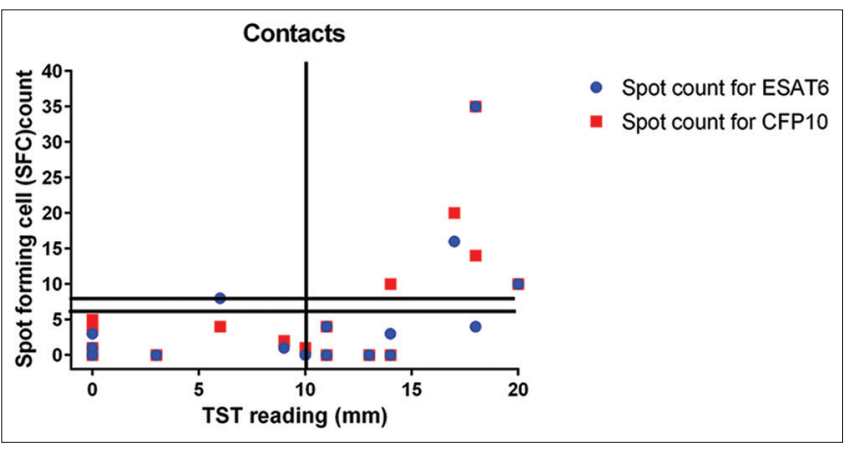

Figure 3: Scatter plots of Tuberculin Skin test reading $(\mathrm{mm})$ vs Spot forming cell (SFC) count for ESAT 6 and CFP10 antigens in control. Reference lines show $10 \mathrm{~mm}$ cut-off for TST positivity (x axis) and SFC count of 6-8 indicating TSPOT.TB positivity (y axis)

not carried out in these subjects due to cost and resource limitations. As mitogen failure was identified as the main reason for indeterminate results, four samples were tested with the quality controlled mitogen reagent requested from the manufacturer as well as the positive control reagent supplied with the kit. These tests showed similar results with failure of mitogen response being seen with both reagents in indeterminate samples and positive mitogen response being seen in other samples. Viability testing showed the cells were still viable in samples that had indeterminate results.

Cost of consumables for a single TSPOT.TB test was approximately LKR 9400.00 (USD 76.00) while a single TST dose cost approximately LKR 200.00 (USD 1.50). Costs associated with transport, storage, labour and overheads were not assessed. As isoniazid prophylaxis is not routinely given in this setting, costs associated with preventive therapy were not estimated.

\section{DISCUSSION}

In this study the presence of DM and cavitation on chest $\mathrm{X}$ ray were significant predictors of T-SPOT.TBpositivity while none of the factors tested were significant predictors of TST positivity. Both tests had similar sensitivity and NPV $(73.5 \%$ and $70.7 \%$ for the T-SPOT.TB and TST respectively). The T-SPOT.TBhad a higher specificity than the TST (75.4 \% and $64.1 \%$ respectively) as well as a higher PPV (72\% vs. $60.4 \%$ ) though the differences were not statistically significant in this study. The tests shows moderate agreement overall, but agreement was only slight when cases alone were considered.

The current use of the IGRAs in Sri Lanka is primarily for diagnosis of active TB in difficult to diagnose cases. As healthy individuals would not have either IGRA or TST done, we included patients being investigated for TB in this study as they better represent the population on whom the test is being used. Significant clinical predictors of T-SPOT. $T B$ positivity were also significant predictors of disease status. This result corresponds to results reported by Pavic et al who showed that cavitatory lesions in adult patients were significantly associated with IGRA positivity in their children. ${ }^{19}$ Interestingly, the TST result was not associated with these factors.

The sensitivity estimate of the IGRA in this study $(73.5 \%)$ is similar to that of studies done in the Gambia $(78 \%),{ }^{20}$ India $(77 \%)^{21}$ and South Africa $(75 \%)^{22}$ but lower than that reported in Taiwan $(83 \%)^{23}$ and Korea $(92 \%) .^{24}$ A meta analysis of 73 studies published by Dai et al showed that the T-SPOT.TBassay had a sensitivity of $88 \%$ in studies done in China and a sensitivity of $90 \%$ in nonChinesestudies. ${ }^{2}$ A meta-analysis of studies done in low and middle income countries by Metcalf et al, showed 
that the pooled sensitivity of the T-SPOT.TBwas $88 \%{ }^{25}$ Studies done in low incidence settings gave sensitivity of $90 \%$ for the TSPOT.TB. ${ }^{26}$

In Chinese studies the specificity was $89 \%$ while in nonChinese studies the value was $74 \%{ }^{2}$ Metcalf et al showed that the pooled specificity based on studies done in low and middle income countries was $61 \%$ for the T-SPOT.TB. ${ }^{25}$ Though in our study a comparatively higher specificity was obtained it was lower than that seen in other low burden BCG vaccinated populations, where specificity of $86-100 \%$ for the T-SPOT.TBhas been reported. ${ }^{26,27}$

The sensitivity/specificity of the TST test using $10 \mathrm{~mm}$ as the cut-off showedits limited use as a screening test, (sensitivity of $70 \%$ and NPV of $73 \%$ ) and a poor value as diagnostic test (specificity of $64 \%$ and PPV of $60 \%$ ). When compared to the results of a meta-analysis done by Pai et al, $70 \%$ sensitivity falls within the $95 \%$ CI of almost all the sensitivities seen in these studied. ${ }^{26}$ The difference between T-SPOT.TBand TST sensitivity is similar to that reported in other moderate-high incidence countries. ${ }^{25}$ The specificity of the TST in our study $(64 \%)$ is similar to that seen in other BCG vaccinated populations $(59 \%$, 95\% CI $46-73 \%)^{26}$ though significantly lower than that in non-BCG vaccinated populations $\left(97 \%\right.$, 95\% CI 95-99\%). ${ }^{26}$ Ongoing antigenic stimulus by both MTB and other mycobacterial antigens in the population probably results in positive TST reactions.

In contact screening test for LTBI, both the T-SPOT.TBand the TST gave statistically similar positivity rates with 53.8\% TST positivity and $26 \%$ IGRA positivity. The TST positive rate is similar to that reported in other low and middle income countries, where household contacts had a 40-50\% positivity rate. ${ }^{28,29}$ The T-SPOT.TBpositivity rate was lower than reported in household contacts in India (48-53\%) 29,30 and Gambia $(40 \%)^{31}$ but was similar to that reported in a Swiss study where $20 \%$ of close contacts were T-SPOT. TBpositive. ${ }^{32}$ Neither test correlated with exposure factors but as the sample size used here was small this conclusion needs confirmation. TST positive/T-SPOT.TBnegative discordant results were seen though the converse (TST negative T-SPOT.TBpositive) was not seen in this group of contacts. T-SPOT.TBpositivity was seen mainly in contacts with highly positive $(>15 \mathrm{~mm})$ TST results. Based on present results, there is no advantage of doing the IGRA over the TST in contact screening in the local population.

The rate of indeterminate results in this study (21\%) is relatively high, though a few other studies have reported similarly high rates, usually in HIV infected patients. ${ }^{25}$ The poor response to the mitogen seen in cases with active TB is similar to the results described elsewhere. ${ }^{33}$
Procedural problems that could have caused these results were investigated but none were found. As poor response to mitogen control was the major cause for indeterminate results, this factor plays an important role in deciding the use of this test locally. Given the high cost of the test, the high rate of indeterminate results is a major reason not to recommend this test for diagnostic purposes in Sri Lanka.

Limitations in this study include the low sample number in the contacts group as well as not repeating the T-SPOT. TBtest on samples when indeterminate or discordant results were found. Follow up studies and repeat testing to look for conversions and reversions of IGRA results was not done as test kits were limited. As random sampling was not used these results may not be generalizable. Smear negative cases were not included, and low risk healthy individuals were not included as a second control group. The primary reason for these limitations in study design was the high cost of the assay.

\section{CONCLUSION}

There was no significant advantage of using the T-SPOT. TBassay as a diagnostic or screening test in the studied Sri Lankan adult population and high cost and high indeterminate result rate makes this test impractical for general use in the local setting.

\section{ACKNOWLEDGEMENTS}

Funding from National Science Foundation of Sri Lanka (research grant RG/HS/2011/08 and equipment grant EQ/2011/25) is gratefully acknowledged. Prof S. Samitha is gratefully acknowledged for assistance with statistical analysis and interpretation.

Part of this data was presented at the $19^{\text {th }}$ Congress of the Asia Pacific Society for Respirology (13-16 ${ }^{\text {th }}$ November 2014) as an oral presentation.

\section{REFERENCES}

1. Denkinger CM, Dheda K and Pai M. Guidelines on interferon- $Y$ release assays for tuberculosis infection: Concordance, discordance or confusion? Clin Microbiol Infect. 2011;17:806-814.

2. Dai $Y$, Feng $Y, X u R, X u$ W, Lu W and Wang J. Evaluation of interferon-gamma release assays for the diagnosis of tuberculosis : An updated meta-analysis. Eur J Clin Microbiol Infect Dis 2012;31:3127-3137.

3. Rangaka MX, Wilkinson K, Glynn JR, Ling D, Menzies D, Mwansa-Kambafwile J, et al. Predictive value of interferon- $\gamma$ release assays for incident active tuberculosis: A systematic review and meta-analysis. Lancet Infect Dis 2012;12:45-55.

4. Vesenbeckh SM, Schönfeld N, Mauch H, Bergmann T, Wagner $S$ and Bauer TT. The use of interferon gamma release 
assays in the diagnosis of active tuberculosis. Tuberc Res Treat 2012;768723.

5. Xiao-min HU, Jian LIU, Long LEI and Wen YIN. Clinical usefulness of T-SPOT. TB in the diagnosis tuberculosis in emergency patients. BMC Emerg Med 2012;12:A7.

6. Denkinger CM, Pai M, Patel M and Menzies D. Gamma interferon release assay for monitoring of treatment response for active tuberculosis: An explosion in the spaghetti factory. J Clin Microbiol 2013;51:607-610.

7. Harari A, Vallelian F, Meylan PR and Pantaleo G. Functional Heterogeneity of Memory CD4 T Cell Responses in Different Conditions of Antigen Exposure and Persistence. J Immunol 2005;174:1037-1045.

8. Millington KA, Innes JA, Hackforth S, Hinks TSC, Deeks JJ and Dosanjh DPS. Dynamic Relationship between IFN- and IL-2 Profile of Mycobacterium tuberculosis-Specific T Cells and Antigen Load. J Immunol 2007;178:5217-5226.

9. Pai M, Denkinger CM, Kik SV, Rangaka MX, Zwerling A and Oxlade O. Gamma Interferon Release Assays for Detection of Mycobacterium tuberculosis Infection. Clin Microbiol Rev 2014;27:3-20.

10. Use of interferon-gamma release assays in support of TB diagnosis. Stockholm: Europaen Center for Disease Control. 2011.

11. Mazurek GH. Updated Guidelines for Using Interferon Gamma Release Assays to Detect Mycobacterium tuberculosis Infection - United States. Centers for Disease Control and Prevention. Morbidity and Mortality Weekly Report. 2010 June (RR05);1-25.

12. Menzies D, editor. Canadian Tuberculosis Standards. $7^{\text {th }}$ Editio. Center for Communicable Disease and Infection Control, Public Health Agency of Canada. 2014.

13. Trajman A, Steffen RE and Menzies D. Interferon-Gamma Release Assays versus Tuberculin Skin Testing for the Diagnosis of Latent Tuberculosis Infection: An Overview of the Evidence. Pulm Med2013:601737. http://www.ncbi.nlm.nih.gov/pmcl articles/PMC3582085.

14. Lee $E$ and Holzman RS. Evolution and current use of the tuberculin test. Clin Inectious Dis 2002;34:365-370.

15. Wang L, Turner MO, Elwood RK, Schulzer M and Fitzgerald JM. A meta-analysis of the effect of Bacille Calmette Guerin vaccination on tuberculin skin test measurements. Thorax 2002;57:804-809.

16. Frieden TR, editor. Toman's Tuberculosis. Case Detection, Treatment and Monitoring - Questions and Answers. $2^{\text {nd }}$ Edition. World Health Organization 2004. http://whqlibdoc.who.int/ publications/2004/9241546034.pdf.

17. Global Tuberculosis Report 2013. World Health Organization Geneva, Switzerland: WHO/HTM/TB/2013.11; 2013. http://apps. who.int/iris/bitstream/10665/91355/1/9789241564656_eng.pdf

18. General manual for tuberculosis control. $2^{\text {nd }}$ edition. National Programme for Tuberculosis Control and Chest Diseases. Ministry of Health, Sri Lanka;. 2005.

19. Pavić I, Topić R, Raos M, Aberle N and Dodig S. Interferon-y release assay for the diagnosis of latent tuberculosis in children younger than 5 years of age. Pediatr Infect Dis J 2011;30:866-870.
20. Adetifa IMO, Lugos MD, Hammond A, Jeffries D, Donkor S and Adegbola R. Comparison of two interferon gamma release assays in the diagnosis of Mycobacterium tuberculosis infection and disease in The Gambia. BMC Infect Dis 2007;7:122.

21. Pai M, Joshi R, Bandyopadhyay $M$, Narang $P$, Dogra $S$ and Taksande B. Sensitivity of a Whole-Blood Interferon-Gamma Assay Among Patients with Pulmonary Tuberculosis and Variations in T-Cell Responses During Anti-Tuberculosis Treatment. Infection 2007;35:98-104.

22. Tsiouris SJ, Coetzee D, Toro PL, Austin J, Stein Z and ElSadr W. Sensitivity analysis and potential uses of a novel gamma interferon release assay for diagnosis of tuberculosis. J Clin Microbiol 2006;44:2844-2850.

23. Lai C-C, Tan C-K, Lin S-H, Liao C-H, Huang Y-T and Hsueh P-R. Diagnostic performance of whole-blood interferon-y assay and enzyme-linked immunospot assay for active tuberculosis. Diagn Microbiol Infect Dis 2011;71:139-143.

24. Kang YA, Lee HW, Hwang SS, Um S-W, Han SK and Shim Y-S. Usefulness of whole-blood interferon-gamma assay and interferon-gamma enzyme-linked immunospot assay in the diagnosis of active pulmonary tuberculosis. Chest 2007;132:959-965.

25. Metcalfe JZ, Everett CK, Steingart KR, Cattamanchi A, Huang L and Hopewell PC. Interferon-y release assays for active pulmonary tuberculosis diagnosis in adults in low- and middleincome countries: Systematic review and meta-analysis. J Infect Dis 2011;204:S1120-S1129.

26. Pai M, Zwerling A and Menzies D. Systematic Review : T-Cell based Assays for the Diagnosis of Latent Tuberculosis Infection: An Update. Ann Intern Med Rev 2008;149:177-184.

27. Dheda K, van Zyl Smit R, Badri M and Pai M. T-cell interferongamma release assays for the rapid immunodiagnosis of tuberculosis: Clinical utility in high-burden vs. low-burden settings. Curr Opin Pulm Med 2009;15:188-200.

28. Fox GJ, Barry SE, Britton WJ and Marks GB. Contact investigation for tuberculosis: A systematic review and metaanalysis. Eur Respir J 2013;41:140-156.

29. Pai $M$, Joshi $R$, Dogra $S$ and Zwerling $A$. T-cell assay conversions and reversions among household contacts of tuberculosis patients in rural India. Int $\mathrm{J}$ Tuberc Lung Dis 200913:84-92.

30. Kashyap RS, NayakAR, Gaherwar HM, Husain A, Shekhawat SD and Jain RK. Latent TB Infection Diagnosis in Population Exposed to TB Subjects in Close and Poor Ventilated High TB Endemic Zone in India. PLoS One 2014;9:e89524.

31. Hill PC, Brookes RH, Fox A, Jackson-Sillah D, Jeffries DJ and Lugos MD. Longitudinal assessment of an ELISPOT test for Mycobacterium tuberculosis infection. PLoS Med 2007;4:e192.

32. Zellweger J-P, Zellweger A, Ansermet S, de Senarclens B and Wrighton-Smith P. Contact tracing using a new T-cell-based test: Better correlation with tuberculosis exposure than the tuberculin skin test. Int J Tuberc lung Dis 2005;9:1242-1247.

33. Feng $J$, Huang $S$, Lee $M$, Ting $W$, Chen $Y$ and Lin $Y$. Characteristics of IFN- $y$ responses in IGRA among pulmonary TB suspects in a TB-endemic area. Diagn Microbiol Infect Dis 2013;77:46-52.

\section{Authors Contribution:}

CR - was involved in planning the study, laboratory work, data analysis and drafting manuscript. VT - was involved in planning the study and manuscript revision and approval. DM - was involved in planning the study, clinical decision making and data collection. ND - was involved in planning to study. AK - was involved in laboratory work and data analysis.

Source of Funding: Grant from National Science Foundation of Sri Lanka (RG/HS/2011/08 and EQ/2011/25), Conflict of Interest: None declared. 